'Fundação Oswaldo Cruz (Fiocruz), Centro de Pesquisa Aggeu Magalhães - Recife (PE), Brasil. gracasborges@gmail.com

\section{O padrão de consumo de álcool entre as usuárias das Unidades de Saúde da Família no município do Recife (PE)}

\author{
The pattern of alcohol consumption among the users of the Family \\ Health Units in the municipality of Recife (PE)
}

Maria das Graças Borges da Silva', Tereza Maciel Lyra', George Tadeu Diniz'

DOI: 10.1590/0103-1104201912214

RESUMO Pesquisas internacionais e nacionais apontam o aumento expressivo de mulheres com consumo abusivo de álcool, e este consumo gera danos sociais, psicológicos e biológicos em suas vidas. O objetivo do presente estudo foi avaliar os padrões do consumo de álcool por mulheres cadastradas nas Unidades de Saúde da Família no município do Recife (PE). Trata de um estudo exploratório descritivo de abordagem quantitativa, que provém de uma pesquisa original, através de uma amostra definida de 864 mulheres com idades iguais ou superiores a 18 anos. Utilizou um questionário sociodemográfico e o Teste para Identificação de Problemas Relacionados ao uso do Álcool. Os padrões de riscos encontrados entre as participantes foram: $57,9 \%$ não bebem; $23,7 \%$ bebem de baixo risco; $11,9 \%$ bebem de médio risco; e 6,3\% bebem de alto risco; predominantemente mulheres jovens, na faixa etária de 18 a 39 anos; sem trabalho e com ensino fundamental. Verificou-se que 54,93\% faziam uso abusivo de bebidas alcoólicas em dia típico e 22,93\% sofreram ocorrência de apagão alcoólico. Esses resultados evidenciam a importância da elaboração de estratégias de cunho preventivo e da redução de danos diante do beber, para a assistência à saúde da mulher.

PALAVRAS-CHAVE Saúde. Mulher. Etanol. Grau de risco. Medicina preventiva.

ABSTRACT International and national surveys point to the significant increase of women with alcohol abuse, and this consumption generates social, psychological and biological damages in their lives. The objective of this study was to evaluate the patterns of alcohol consumption among women enrolled in the Family Health Units in Recife (PE). It is an exploratory descriptive study with a quantitative approach, which comes from an original survey through a defined sample of 864 women aged 18 years or older. It used a sociodemographic questionnaire and the Test for Identification of Problems Related to the use of Alcohol. The risk standards found among participants were $57.9 \%$ do not drink; $23.7 \%$ drink at low risk; $11.9 \%$ drink at medium risk; and 6.3\% drink at high risk; predominantly young women, in the age group 18 to 39; without work and elementary school. It was verified that $54.93 \%$ made abusive use of alcoholic beverages in a typical day and $22.93 \%$ suffered an alcoholic blackout. These results evidence the importance of elaborating preventive and harm reduction strategies in relation to drinking, for women's health care.

KEYWORDS Health. Woman. Ethanol. Risk grade. Preventive medicine. 


\section{Introdução}

Estudos epidemiológicos de âmbito internacional e nacional apontam o crescimento do número de mulheres consumidoras de substâncias psicoativas, sobretudo o álcool. Além disso, apontam que o número de mulheres com uso abusivo de álcool triplicou nos últimos anos, em âmbito mundial, inclusive no Brasil1,2.

Atualmente, considera-se uso abusivo de álcool a ingestão de quatro ou mais doses, para mulheres, e cinco ou mais doses, para homens, em uma mesma ocasião. Acima destas quantidades, o indivíduo tem maior risco de apresentar problemas relacionados ao consumo de álcool. Internacionalmente, tal prática é denominada binge drinking. Concebe-se como dose de bebida alcoólica uma dose de bebida destilada, uma lata de cerveja ou uma taça de vinho, ${ }^{2,3}$.

O álcool é uma droga psicotrópica, que atua no sistema nervoso central e provoca mudança de comportamento. É uma substância lícita, com ampla aceitação social de seu consumo, para ambos os sexos ${ }^{2,3}$.

No contexto internacional, a Organização Mundial da Saúde (OMS) divulgou, em 2018, um relatório sobre o consumo de álcool no mundo, e também noticiou alguns avanços alcançados pelas políticas de Redução do Uso Abusivo do Álcool adotadas em 194 países. Os dados apontaram um maior percentual de mortes entre os homens do que entre as mulheres, por causas relacionadas ao álcool. Além disso, observaram o aumento constante do uso abusivo de álcool entre as mulheres jovens, em âmbito mundial. No Brasil, o álcool esteve associado a $69,5 \%$ e $42,6 \%$ dos casos de cirrose hepática, e a $36,7 \%$ e $23 \%$ dos acidentes de trânsito, entre homens e mulheres, respectivamente, em ambos os índices. Especificamente em relação aos transtornos relacionados ao uso de álcool, estima-se que 4,2\% (mulheres: 1,6\%; homens: 6,9\%) dos brasileiros preenchem critérios para abuso ou dependência'.

No Brasil, anualmente, é efetivada uma pesquisa sobre Vigilância de Fatores de Risco e Proteção para Doenças Crônicas, através de inquérito telefônico, denominada Vigitel, realizado com pessoas com 18 anos ou mais, nas capitais estaduais e no Distrito Federal. Em 2017, a pesquisa apontou que a prevalência média de risco no consumo de bebidas alcoólicas, nos últimos 30 dias, havia alcançado $19,1 \%$, sendo maior para os homens $(27,1 \%)$ do que para as mulheres $(12,2 \%)^{4}$.

O consumo abusivo de álcool entre as mulheres do município do Recife (PE) é equivalente à média nacional (12,1\%). Em ambos os sexos, a frequência de consumo abusivo de bebidas alcoólicas tendeu a diminuir com a idade e a aumentar com a escolaridade ${ }^{4}$.

A mulher que apresenta contínuo consumo abusivo de álcool vem sendo estigmatizada pela sociedade, e isto influi negativamente sobre seu estado de saúde, tornando-o mais difícil - ou mesmo, impedindo - seu acesso aos serviços de saúde, o que acaba levando-a a procurar tratamento com menos assiduidades do que os homens, acarretando-lhes mais comprometimentos ao longo do tempo ${ }^{5-7}$. Além do estigma social, outras implicações são observadas pelo consumo abusivo de bebidas alcoólicas entre mulheres, que podem estar associadas a diversos problemas de saúde, tais como: prática de sexo sem proteção, síndrome pré-menstrual, câncer de mama, problemas de fertilidade, entre outros ${ }^{5-7}$.

A maior vulnerabilidade entre as mulheres ocorre porque a ingestão de bebidas alcoólicas passa por diferentes caminhos daqueles percorridos pelos homens. Biologicamente, elas são menos tolerantes ao álcool do que eles. No organismo feminino, o álcool atinge concentrações sanguíneas mais altas, com as mesmas doses, devido à menor quantidade de água corporal e em função de maior quantidade de gordura, associada a uma menor quantidade de enzimas que metabolizam o álcool. Esta característica reflete o fato de que as mulheres geralmente só precisam de metade das doses ingeridas pelos homens para se intoxicarem, isto sempre considerando peso e altura ${ }^{5-7}$. 
Os problemas relacionados ao álcool entre as mulheres têm sido frequentemente negligenciados, resultando em possíveis negações, ao invés de cuidados ${ }^{7}$. Entretanto, elas, que eram tradicionalmente consideradas consumidoras leves de bebidas alcoólicas, estão alcançando padrões de consumo abusivo equivalentes aos dos homens ${ }^{2,6,7}$.

Estudos apontam que o uso abusivo de bebidas alcoólicas é um dos quatro principais fatores de risco para as doenças não transmissíveis que são suscetíveis de modificação comportamental e prevenção ${ }^{\mathbf{1}, \mathbf{7}}$. Neste contexto, a OMS e o Ministério da Saúde (MS) orientam que os profissionais das Unidades Básicas de Saúde (UBS) adotem na rotina de cuidado, a detecção do padrão de uso de álcool, tanto nos homens quanto nas mulheres, a fim de promoverem ações preventivas, estratégias de redução de danos e eventuais encaminhamentos ao tratamento especializado ${ }^{8}$. Além disto, espera-se que esses profissionais reconheçam a realidade dos usuários em seus contextos culturais, a fim de que possam oferecer-lhes outros referenciais e subsídios que gerem mudanças de conduta e reduzam os possíveis danos diante do uso de álcool ${ }^{8}$.

Diante do elevado consumo de bebidas alcoólicas e considerando a importância dos estudos epidemiológicos para o direcionamento de políticas públicas que visem à redução dos problemas decorrentes deste uso, desenvolveu-se este estudo, com o objetivo de identificar o padrão de consumo de bebidas alcoólicas em mulheres que utilizam o Sistema Único de Saúde (SUS), acompanhadas pelas Unidades de Saúde da Família (USF) da Secretaria Municipal de Saúde do Recife, no estado de Pernambuco.

Vale ressaltar que, embora o presente estudo se situe no município do Recife (PE), grande metrópole regional, considera-se que seus achados contribuirão tanto para o estado da arte quanto para aprofundar o debate sobre a problemática do consumo de bebidas alcoólicas por mulheres, nos demais municípios do estado e em outras regiões do País.

\section{Procedimentos metodológicos}

Tratou-se de um estudo quantitativo, de caráter exploratório descritivo e delineamento transversal, realizado nas 20 USF de um distrito sanitário da Secretaria Municipal de Saúde do Recife, em uma instituição pública mantida pelo SUS.

No total, 864 mulheres com idades iguais ou superiores a 18 anos participaram da pesquisa, tendo sido escolhidas de forma aleatória.

A coleta de dados ocorreu no período de março a maio de 2017. Foram utilizados dois instrumentos para isso: o primeiro, elaborado exclusivamente para este estudo, foi definido pela necessidade de caracterização da amostra e constou de um questionário sociodemográfico, com questões referentes a idade, religião, escolaridade, situação conjugal, trabalho, moradia e renda familiar. O segundo foi o Teste para Identificação de Problemas Relacionados ao Uso do Álcool (Audit), que é um questionário validado pela OMS e adotado pelo Brasil, no intuito de identificar os diversos padrões de uso de álcool. A aplicação dos dois instrumentos foi efetivada pela primeira autora deste artigo.

O teste Audit contém dez questões relacionadas ao uso de álcool e é focado nos últimos 12 meses deste consumo. É formado em três dimensões conceituais, que avaliam quantidade e frequência de consumo de álcool (itens 1 a 3), ocorrência de sintomas de dependência (itens 4 a 6) e consequências relacionadas ao consumo excessivo de bebidas alcoólicas (itens 7 a 10)9,10.

Na classificação das participantes frente ao consumo de bebidas alcoólicas foi realizada uma readaptação das zonas de risco. Assim, elas foram classificadas como abstêmios; zona 0, quando a pontuação do Audit foi igual a zero; beber de baixo risco, representando a zona I (1 a 7 pontos); beber de médio risco, correspondente à zona II (8 a 15 pontos); e beber de alto risco, correspondendo à zona III (16 ou mais pontos) ${ }^{10}$. 
A identificação do nível de risco abre caminho para que o profissional da Atenção Primária à Saúde (APS) possa oferecer orientações personalizadas, focadas no padrão de consumo individual12,13. Sua finalidade é detectar precocemente os níveis de risco em que se encontram os usuários, permitindo aos profissionais da área da saúde a visualização de pessoas que necessitam reduzir ou cessar o uso de bebidas alcoólicas. Assim, os profissionais da APS podem adotar, em seus atendimentos, medidas preventivas que auxiliem na redução dos prejuízos associados ao álcool9,10.

Realizou-se a análise estatística descritiva dos dados, organizando-os a fim de apresentar um conjunto de dados, com vistas a obter informações e conhecer as características de um determinado grupo. Esta modalidade torna possível o conhecimento das variáveis analisadas, fornecendo importantes direções a serem seguidas em estudos futuros" ${ }^{11}$.

Na observação do comportamento, comparativa, entre as variáveis qualitativas, adotou-se uma análise categórica, utilizando-se o Teste Qui-quadrado de Pearson, e, quando necessário, o Teste Exato de Fisher. Todas as conclusões foram tomadas com nível de significância de $5 \%$. O software R (R Development Core Team, 2016) foi utilizado na avaliação dos resultados do estudo. As participantes foram analisadas por grupos etários: 18 a 29 anos; 30 a 39 anos; 40 a 49 anos; e 50 anos ou mais.

$\mathrm{O}$ estudo seguiu normas e procedimentos éticos de acordo com a Resolução n ${ }^{\circ}$ 466/2012, tendo sido aprovado pelo Comitê de Ética em Pesquisa do Centro de Pesquisas Aggeu Magalhães (CPqAM)/Fundação Oswaldo Cruz (Fiocruz Pernambuco), processo ${ }^{\circ}{ }^{0}$ 1.594.177.

\section{Resultados e discussões}

O perfil sociodemográfico das 864 participantes revelou que a idade média das mulheres foi de 39 anos; com mínima de 18 e máxima de 74. Já as mulheres com idade inferior a 50 anos representaram $68 \%$ da amostra.

A maioria das participantes (92\%) seguia alguma religião; $74,1 \%$ moravam em casa própria; $68 \%$ eram do lar (domésticas); $22,1 \%$ eram chefes de família. Em relação à escolaridade, a maioria (87,3\%) possuía ensino fundamental, e apenas 2,8\% delas tinham curso superior.

Os resultados do teste Audit, em relação às questões 1, 2 e 3 - que correspondem à primeira dimensão, avaliaram a frequência do consumo de álcool (tabela 1). Apontaram que $57,9 \%$ não bebiam e $42 \%$ bebiam. Foi observado que $8,8 \%$ bebiam de duas a três vezes por semana, e $2 \%$ bebiam quase diariamente.

No entanto, é importante destacar que, no que se refere à quantidade do consumo de álcool em um dia típico, o resultado apontou que $11,25 \%$ beberam de cinco a seis doses; $4,6 \%$ consumiram de sete a nove doses; e 39,2\% beberam dez ou mais doses. Revelou-se que $54,93 \%$ faziam uso abusivo de álcool. Adverte-se, no entanto, que a OMS preconiza que mais de quatro doses de consumo, para mulheres, já podem configurar uso abusivo de bebidas alcoólicas.

Tabela 1. Distribuição das respostas das questões 1, 2 e 3 do Teste Audit das participantes. Recife (PE), Brasil, 2017

\begin{tabular}{lrr}
\hline Teste de Identificação de Transtornos Devido ao Uso do Álcool (Audit) & N & $\%$ \\
\hline Q1 - Com que frequência você toma bebidas alcoólicas? & $501^{\star}$ & 57,99 \\
\hline Nunca & 202 & 23,38 \\
Mensalmente ou menos & 67 & 7,75 \\
De 2 a 4 vezes por mês & 76 & 8,8 \\
De 2 a 3 vezes por semana & 18 & 2,08 \\
4 ou mais vezes por semana & 18 \\
\hline
\end{tabular}


Tabela 1. (cont.)

\begin{tabular}{|c|c|c|}
\hline \multicolumn{3}{|c|}{ Q2 - Nas ocasiões em que bebe, quantas doses você consome tipicamente? } \\
\hline 1 ou 2 & 75 & 20,6 \\
\hline 3 ou 4 & 88 & 24,18 \\
\hline 5 ou 6 & 41 & 11,26 \\
\hline 7,8 ou 9 & 17 & 4,67 \\
\hline 10 ou mais & 143 & 39,29 \\
\hline \multicolumn{3}{|c|}{ Q3 - Com que frequência você toma 'seis ou mais doses' de uma vez? } \\
\hline Nunca & 161 & 44,08 \\
\hline Menos do que uma vez ao mês & 77 & 21,21 \\
\hline Mensalmente & 43 & 11,85 \\
\hline Semanalmente & 75 & 20,66 \\
\hline Todos ou quase todos os dias & 8 & 2,2 \\
\hline
\end{tabular}

Já as questões de 4 a 6 do teste Audit, que correspondem à segunda dimensão, investigaram os possíveis sintomas relacionados à dependência, trazendo à tona um aspecto preocupante (tabela 2).

Um desses aspectos foi a quantidade de tentativas de beber poucas doses, que algumas mulheres fizeram, sem conseguir. O resultado apontou que o beber compulsivo foi de $14,61 \%$. Para 9,9\% delas, isso ocorreu pelo menos uma vez ao mês.

Outro aspecto preocupante foi a elevada prevalência (18,73\%), entre as participantes, daquelas que deixaram de realizar suas atividades planejadas para um determinado dia devido ao consumo de bebidas no dia anterior. Também foi observada a necessidade de ingestão de bebida alcoólica, logo pela manhã, por parte de $6,61 \%$ das mulheres, ainda que tenham passado a noite anterior bebendo.

As respostas das participantes obtidas nas questões de 4 a 6 sinalizaram um aspecto preocupante no que se refere aos sintomas de uma possível dependência de álcool.

Tabela 2. Distribuição das respostas das questões 4, 5 e 6 do Teste Audit das participantes. Recife (PE), Brasil, 2017

\begin{tabular}{lrr}
\hline Teste de Identificação de Transtornos Devido ao Uso do Álcool (Audit) & N & $\%$ \\
\hline Q4 - Quantas vezes, ao longo dos últimos 12 meses, você achou que não conseguiria parar de beber, uma vez tendo \\
começado? & 298 & 82,09 \\
\hline Nunca & 36 & 9,92 \\
Menos do que uma vez ao mês & 12 & 3,31 \\
Mensalmente & 12 & 3,31 \\
Semanalmente & 5 & 1,38 \\
\hline Todos ou quase todos os dias & 5
\end{tabular}


Tabela 2. (cont.)

\begin{tabular}{|c|c|c|}
\hline \multicolumn{3}{|c|}{$\begin{array}{l}\text { Q5 - Quantas vezes, ao longo dos últimos } 12 \text { meses, por causa do álcool, não conseguiu fazer o que era esperado de } \\
\text { você? }\end{array}$} \\
\hline Nunca & 295 & 81,27 \\
\hline Menos do que uma vez ao mês & 51 & 14,05 \\
\hline Mensalmente & 10 & 2,75 \\
\hline Semanalmente & 4 & 1,1 \\
\hline Todos ou quase todos os dias & 3 & 0,83 \\
\hline \multicolumn{3}{|c|}{$\begin{array}{l}\text { Q6 - Quantas vezes, ao longo dos últimos } 12 \text { meses, você precisou beber pela manhã para se sentir bem ao longo do } \\
\text { dia, após ter bebido no dia anterior? }\end{array}$} \\
\hline Nunca & 339 & 93,39 \\
\hline Menos do que uma vez ao mês & 11 & 3,03 \\
\hline Mensalmente & 5 & 1,38 \\
\hline Semanalmente & 6 & 1,65 \\
\hline Todos ou quase todos os dias & 2 & 0,55 \\
\hline
\end{tabular}

Fonte: Elaboração própria.

Por fim, a terceira dimensão, composta pelas quatro questões finais do teste Audit, avaliou os problemas recentes relacionados ao consumo de álcool (tabela 3). Observou-se que $22,93 \%$ das mulheres desconheciam o que havia sucedido durante o consumo, sendo notório o apagão alcoólico. Uma parte das entrevistadas $(3,9 \%)$ chegou a cometer atos de violência contra si mesmas.

Ainda foi observado que $83,5 \%$ das participantes não tiveram nenhum tipo de orientação, em âmbito pessoal ou profissional, sobre o beber feminino. Deste modo, pode-se pensar que os profissionais de saúde que atuam nas USF muito pouco têm feito em relação aos cuidados voltados para o consumo de bebidas alcoólicas e às possíveis consequências de doenças orgânicas associadas a este consumo, principalmente ao gênero feminino. O fato de o álcool ser uma substância lícita, de uso aceito e até incentivado pela sociedade, talvez explique a resistência ou o desconhecimento dos profissionais de saúde para lidarem com esta demanda. Estudos de Monteiro et al. ${ }^{6}$ ressaltam que os profissionais de saúde desconhecem os níveis de risco e de danos gerados pelo consumo de bebidas alcoólicas entre as mulheres, tanto no Brasil como em outros países.

Destaque-se que a imagem da mulher, historicamente construída, não está de acordo com a imagem de pessoa usuária de drogas, $\mathrm{o}$ que contribui para o processo de invisibilidade feminina em relação a tal fenômeno e, consequentemente, para uma maior vulnerabilidade em relação a agravos sociais e de saúde, bem como a dependência alcoólica. 


\begin{tabular}{|c|c|c|}
\hline Teste de Identificação de Transtornos Devido ao Uso do Álcool (Audit) & $\mathbf{N}$ & $\%$ \\
\hline \multicolumn{3}{|c|}{ Q7 - Quantas vezes, ao longo dos últimos 12 meses, você se sentiu culpado ou com remorso depois de ter bebido? } \\
\hline Nunca & 274 & 75,48 \\
\hline Menos do que uma vez ao mês & 60 & 16,53 \\
\hline Mensalmente & 15 & 4,13 \\
\hline Semanalmente & 11 & 3,03 \\
\hline Todos ou quase todos os dias & 3 & 0,83 \\
\hline \multicolumn{3}{|c|}{ Q8 - Quantas vezes, ao longo dos últimos 12 meses, você foi incapaz de lembrar o que aconteceu devido à bebida? } \\
\hline Nunca & 279 & 77,07 \\
\hline Menos do que uma vez ao mês & 56 & 15,47 \\
\hline Mensalmente & 11 & 3,04 \\
\hline Semanalmente & 13 & 3,59 \\
\hline Todos ou quase todos os dias & 3 & 0,83 \\
\hline \multicolumn{3}{|c|}{ Q9 - Alguma vez na vida você já causou ferimentos ou prejuízos a você mesmo ou a outra pessoa após ter bebido? } \\
\hline Não & 796 & 92,13 \\
\hline Sim, mas não nos últimos 12 meses & 34 & 3,94 \\
\hline Sim, nos últimos 12 meses & 34 & 3,94 \\
\hline \multicolumn{3}{|c|}{$\begin{array}{l}\text { Q10 - Alguma vez na vida algum parente, amigo, médico ou outro profissional da saúde já se preocupou com o fato } \\
\text { de você beber ou sugeriu que você parasse? }\end{array}$} \\
\hline Não & 715 & 83,53 \\
\hline Sim, mas não nos últimos 12 meses & 54 & 6,31 \\
\hline Sim, nos últimos 12 meses & 87 & 10,16 \\
\hline
\end{tabular}

Fonte: Elaboração própria.

As classificações dos padrões de risco das 864 participantes frente ao consumo de bebidas alcoólicas revelaram que $57,9 \%$ não bebem, ou seja, não apresentam risco; 23,7\% bebem de baixo risco; $11,9 \%$ bebem de médio risco; e 6,3\% bebem de alto risco (tabela 4).

Em relação à idade, as mulheres classificadas como de baixo risco apresentaram distribuição semelhante das mulheres classificadas como sem risco.

Entre as mulheres jovens na faixa etária de 18 a 29 anos, as prevalências apontaram tanto para médio risco (42,5\%) quanto para alto risco (32,7\%). Já entre as mulheres de 30 anos a 39 anos, as prevalências estiveram no médio risco $(22,3 \%)$ e no alto risco $(27,2 \%)$. No entanto, as prevalências mais baixas foram observadas entre as mulheres de 40 anos ou mais, tanto de médio risco quanto de alto risco, com $15,5 \%$ e $14,5 \%$, respectivamente.

A literatura denuncia que o consumo abusivo de álcool entre jovens de ambos os sexos não é um fenômeno recente no Brasil, ou em outros países, o que não dispensa a preocupação e a mobilização das autoridades educacionais e da saúde pública em relação aos cuidados devidos com o tema, dadas as possíveis consequências de tal consumo ${ }^{\mathbf{1}, \mathbf{4}, \mathbf{6}, \mathbf{7}}$.

Outra prevalência alta em todos os padrões de risco foi relativa ao fato de as mulheres não trabalharem, possuindo renda familiar menor do que um salário mínimo. Estudo de Guimarães et al.12 apontou que um dos fatores predisponentes ao uso abusivo de bebidas alcoólicas é a ausência de trabalho.

Os resultados sugeriram, também, certa 
associação entre a variável crença religiosa e o padrão de consumo de bebidas alcoólicas, apontando que o fato de algumas mulheres não terem crença foi associado a padrões de risco. As mulheres estudadas, que não mencionaram religião ou qualquer tipo de crença, apresentaram um aumento de prevalências entre os diferentes níveis de risco (baixo risco, de $8,3 \%$; médio risco, $11,6 \%$; alto risco, $16,3 \%$ ), na medida em que os padrões de risco cresceram. Assim, a religiosidade - ou o fato de terem alguma crença - foi apontada como inibidora do consumo de bebidas alcoólicas. Este efeito vem sendo confirmado na literatura ${ }^{\mathbf{1 3}, \mathbf{1 4}}$.

A prevalência do padrão de alto risco - de $6,3 \%$, entre as avaliadas - foi elevada, maior do que a encontrada em pesquisa realizada especificamente na USF, situada na Zona Oeste do município do Rio de Janeiro (RJ), que utilizou o teste Audit. O estudo avaliou o consumo de bebidas alcoólicas em uma amostra composta por 301 sujeitos, com idades iguais ou superiores a 18 anos. Entre eles, pouco mais da metade $(59,8 \%)$ era composta por mulheres. A porcentagem do consumo de bebidas alcoólicas em padrão de alto risco, entre homens, foi de $9,9 \%$; e entre mulheres, foi de $3,9 \%^{14}$.

A literatura vem acenando uma associação positiva entre a ingestão de álcool e o câncer de mama. A ingestão de álcool é um fator de risco considerável, pois estudos demonstraram que até mesmo seu uso social aumenta os níveis de estrogênio endógeno no organismo, o que pode provocar alterações nas células mamárias e transformá-las em tumores malignos. Ou seja, a ingestão configura um mecanismo potencial para o risco de câncer de mama entre as mulheres. Os resultados do presente trabalho chamam a atenção para a prevalência das participantes que beberam dez ou mais doses em dia típico, que foi de 39,29\%. Dessas, $9 \%$ encontravam-se em baixo risco, $53 \%$ em médio risco e $38 \%$ em alto risco ${ }^{15-18}$.

Nos atendimentos das USF, tanto a literatura internacional quanto a nacional indicaram presença entre $3 \%$ e $10 \%$ de pessoas com problemas relacionados ao álcool nesses serviços, e advertiram que tais usuários se encontravam sem cuidado ou encaminhamento para serviço especializado. Verificou-se, ainda, que os profissionais de saúde tinham dificuldades para identificar os problemas causados pelo uso de álcool nos atendimentos dessa clientela, revelando um possível despreparo daqueles profissionais ${ }^{19,20}$.

Nas raras ocasiões em que há um olhar mais atento para a questão, este tem sido essencialmente voltado para os homens, fato que sugere que os profissionais de saúde acreditam que as mulheres controlam melhor a ingestão de bebidas alcoólicas. Isto vem dificultando a dedicação de um cuidado maior na atenção à saúde da mulher, diante dos danos e prejuízos gerado pelo consumo abusivo de álcool19,20.

Nesse contexto, é fundamental um investimento na educação permanente dos profissionais do SUS, de maneira especial, das equipes de USF. Todavia, uma educação permanente em saúde representa um conjunto de ações essenciais que possibilitam a qualificação dos profissionais responsáveis pelos cuidados ofertados aos usuários, além de permitir a integração da vivência diária com os conteúdos teóricos, para que haja qualidade na execução do trabalho. Esta educação se baseia no problema vivenciado em cada localidade, a fim de resolvê-lo através da oferta de conhecimento e capacitação dos trabalhadores ${ }^{21}$.

Destaque-se que as conquistas sociais e dos direitos femininos não representam apenas vantagens, afinal, a ampliação de direitos implica no aumento de deveres, havendo, portanto, também os aspectos negativos deste novo modo de ser/existir. Assim, os costumes e atitudes que antes eram exclusivamente masculinos começam a fazer parte da realidade feminina, o que inclui o consumo abusivo de álcool7. Com a mudança do papel social feminino, a conduta de beber socialmente, entre mulheres, parece ter julgamento mais ameno em determinadas culturas.

Enfim, os resultados apontaram índice expressivo $(23,75 \%)$ de consumo de álcool entre as participantes classificadas em padrão de 
baixo risco, sendo indicadas ações preventivas e educativas sobre o consumo de bebidas alcoólicas e suas consequências para a saúde.

Outro índice que chamou a atenção foi o referente tanto às classificadas em médio risco $(11,92 \%)$ quanto às classificadas em alto risco (6,3\%), diante do consumo de álcool. Tendo sido tais padrões considerados potencialmente danosos à saúde, é importante que as USF adotem estratégias preventivas e de redução de danos, se necessário, encaminhando-as a um Centro de Atenção Psicossocial - Álcool e outras Drogas (CAPSad).

Neste contexto, é fundamental trabalhar a prevenção na perspectiva da abordagem de redução de danos diante dos padrões de risco no uso de álcool, compreendendo que o melhor caminho para lidar com o padrão de consumo de bebidas alcoólicas não é o de decidir e definir pelos usuários quais são as condutas mais adequadas e corretas, $\mathbf{7 2 2}$.
Muito diferente disto, é construir, junto com eles, possibilidades de escolhas mais confiáveis e livres, diminuindo-lhes os riscos e as vulnerabilidades.

Atualmente, a redução de danos constitui-se como uma estratégia de saúde pública a ser adotada globalmente, de acordo com a OMS1. Porém, a nova Política Nacional sobre Drogas (PNAD), instituída através do Decreto ${ }^{\circ} 9.761$, de 11 de abril de 2019, aprovou o fim da lógica de redução de danos, priorizando a abstinência dos usuários de drogas ${ }^{23}$. Ressaltando-se que o princípio da abstinência reforça ideias gerais sobre certo e errado e é pautado em dogma moralista, neste sentido, pode-se entender que tal abordagem pode aumentar os danos e os entraves no enfrentamento dessa problemática, configurando-se, de fato, como um retrocesso, e vai na contramão da lógica da redução de danos já instituída na maioria das nações desenvolvidas.

Tabela 4. A associação do perfil sociodemográfico com o grau de risco no uso de álcool nas mulheres estudadas nas USF. Recife (PE), Brasil, 2017

\begin{tabular}{|c|c|c|c|c|c|c|c|c|c|}
\hline \multirow{3}{*}{$\begin{array}{l}\text { Variáveis } \\
\text { sociodemográficas }\end{array}$} & \multicolumn{8}{|c|}{ Zona (Grau de Risco) } & \multirow{3}{*}{$\begin{array}{r}\text { Valor } \\
\text { de P }\end{array}$} \\
\hline & \multicolumn{2}{|c|}{ Sem Risco } & \multicolumn{2}{|c|}{ Baixo Risco } & \multicolumn{2}{|c|}{ Médio Risco } & \multicolumn{2}{|c|}{ Alto Risco } & \\
\hline & $\mathbf{N}$ & $\%$ & $\mathrm{~N}$ & $\%$ & $\mathbf{N}$ & $\%$ & $\mathbf{N}$ & $\%$ & \\
\hline Total & 501 & 57,99 & 205 & 23,73 & 103 & 11,92 & 55 & 6,37 & \\
\hline \multicolumn{10}{|l|}{ Idade (anos) } \\
\hline 1) $50+$ & 167 & 33,33 & 72 & 35,12 & 23 & 22,33 & 15 & 27,27 & 0,0067 \\
\hline 2) $18-29$ & 125 & 24,95 & 41 & 20 & 43 & 41,75 & 18 & 32,73 & \\
\hline 3) $30-39$ & 96 & 19,16 & 43 & 20,98 & 21 & 20,39 & 14 & 25,45 & \\
\hline 4) $40-49$ & 113 & 22,55 & 49 & 23,9 & 16 & 15,53 & 8 & 14,55 & \\
\hline \multicolumn{10}{|l|}{ Religião } \\
\hline 1) Com & 474 & 94,61 & 187 & 91,22 & 91 & 88,35 & 46 & 83,64 & 0,0066 \\
\hline 2) Sem & 27 & 5,39 & 18 & 8,78 & 12 & 11,65 & 9 & 16,36 & \\
\hline \multicolumn{10}{|l|}{ Estado civil } \\
\hline 1) Casado/União & 347 & 69,26 & 139 & 67,8 & 55 & 53,4 & 32 & 58,18 & 0,0231 \\
\hline 2) Viúvo/Separado & 32 & 6,39 & 18 & 8,78 & 8 & 7,77 & 3 & 5,45 & \\
\hline 3) Solteiro & 122 & 24,35 & 48 & 23,41 & 40 & 38,83 & 20 & 36,36 & \\
\hline
\end{tabular}


Tabela 4. (cont.)

\begin{tabular}{|c|c|c|c|c|c|c|c|c|c|}
\hline \multicolumn{10}{|l|}{ Escolaridade } \\
\hline 0) Superior & 9 & 1,8 & 11 & 5,37 & 4 & 3,88 & 0 & 0 & 0,0002 \\
\hline 1) Fundamental & 316 & 63,07 & 113 & 55,12 & 60 & 58,25 & 48 & 87,27 & \\
\hline 2) Médio & 176 & 35,13 & 81 & 39,51 & 39 & 37,86 & 7 & 12,73 & \\
\hline \multicolumn{10}{|l|}{ Economicamente } \\
\hline 1) Ativo & 137 & 27,35 & 76 & 37,07 & 46 & 44,66 & 16 & 29,09 & 0,0016 \\
\hline 2) Inativo & 364 & 72,65 & 129 & 62,93 & 57 & 55,34 & 39 & 70,91 & \\
\hline \multicolumn{10}{|l|}{ Residência } \\
\hline 1) Própria & 364 & 72,65 & 150 & 73,17 & 82 & 79,61 & 40 & 72,73 & 0,5914 \\
\hline 2) Alugada & 92 & 18,36 & 41 & 20 & 17 & 16,5 & 12 & 21,82 & \\
\hline 3) Outro & 45 & 8,98 & 14 & 6,83 & 4 & 3,88 & 3 & 5,45 & \\
\hline \multicolumn{10}{|c|}{ Composição familiar } \\
\hline 1) $6 \mathrm{ou}+$ & 90 & 17,96 & 36 & 17,56 & 21 & 20,39 & 13 & 23,64 & 0,6932 \\
\hline 2) $\leq 2$ & 303 & 60,48 & 128 & 62,44 & 56 & 54,37 & 28 & 50,91 & \\
\hline 3) 3 a 5 & 108 & 21,56 & 41 & 20 & 26 & 25,24 & 14 & 25,45 & \\
\hline \multicolumn{10}{|l|}{ Chefe da família } \\
\hline 1) Outros & 404 & 80,64 & 159 & 77,56 & 70 & 67,96 & 37 & 67,27 & 0,0101 \\
\hline 2) A Própria & 97 & 19,36 & 46 & 22,44 & 33 & 32,04 & 18 & 32,73 & \\
\hline \multicolumn{10}{|l|}{ Renda Familiar } \\
\hline 1) Um Salário & 180 & 35,93 & 82 & 40 & 29 & 28,16 & 20 & 36,36 & 0,3303 \\
\hline 2) > Um Salário & 85 & 16,97 & 29 & 14,15 & 20 & 19,42 & 13 & 23,64 & \\
\hline 3) < Um Salário & 236 & 47,11 & 94 & 45,85 & 54 & 52,43 & 22 & 40 & \\
\hline
\end{tabular}

Fonte: Elaboração própria.

\section{Considerações finais}

O presente estudo permitiu identificar os padrões de risco do abuso de álcool em mulheres acompanhadas pelas USF. O conhecimento sistematizado deste padrão de risco é crucial para auxiliar o desenvolvimento de políticas públicas direcionadas à saúde da mulher nos diferentes níveis de complexidade da atenção em saúde. As peculiaridades e especificidades do consumo de álcool em mulheres devem ser consideradas na tomada de decisão dos gestores.

Grife-se que a ingestão de bebida alcoólica é parte complementar da vida social. Falar sobre o álcool é também falar sobre a sociedade, pois o ato de beber é um ato social. Isso porque o ser humano sempre buscou, através dos tempos, maneiras de aumentar o seu prazer e diminuir o seu sofrimento.

Tratando-se do consumo de bebidas alcoólicas entre mulheres, esta problemática mantém-se ainda invisível para grande parcela da sociedade e para as próprias políticas públicas, que não priorizam as suas particularidades. É fundamental cuidar e prevenir, adotando a construção de redes sociais que visem à melhoria das condições 
${ }^{*}$ Orcid (Open Researcher and Contributor ID). de vida na perspectiva da Atenção Integral à Saúde da Mulher.

Nesse sentido, sugere-se um aprofundamento na questão para compreender as ações socialmente construídas diante de tal problemática no universo feminino. Além disto, é necessário discutir como e o que fazer para sensibilizar as mulheres em relação aos danos provenientes do consumo abusivo de álcool para suas próprias vidas.

\section{Colaboradores}

Silva MGB (0000-0002-1582-0496)* e Lyra TM (0000-0002-3600-7250)* contribuíram para a concepção, o planejamento, a análise e a interpretação dos dados; revisão crítica do conteúdo; e aprovação da versão final do manuscrito. Diniz GT (0000-0002-9620-2621)* contribuiu para a análise dos dados, a revisão crítica do conteúdo e a aprovação da versão final.

\section{Referências}

1. Organização Mundial da Saúde. Global status report on alcohol and health. 2018. World Health Organization. Global status report on alcohol and health 2018 [internet]. Geneva: World Health Organization; 2018 [acesso em 2018 set 21]. Disponível em: https://www. who.int/substance_abuse/publications/global_alcohol_report/en/.

2. Reisdorfer E, Büchele F, Pires ROM, et al. Prevalence and associated factors with alcohol use disorders among adults: a population-based study in southern Brazil. Rev. bras. de epidemiol. 2012; 15(3):582-594.

3. Solberg LI, Maciose K MV, Edwards NM. Primary care intervention to reduce alcohol misuse. Ranking its health impact and cost effectiveness. Am J Prev Med. 2008; 34(2):143-152.

4. Brasil. Ministério da Saúde, Secretaria de Vigilância em Saúde. Vigitel Brasil 2017: vigilância de fatores de risco e proteção para doenças crônicas por inquérito telefônico. Brasília, DF: Ministério da Saúde; 2018. 130 p.
5. Fortney J, Mukherjee S, Curran G, et al. Factors associated with perceived stigma for alcohol use and treatment among at-risk drinkers. J. behav. health serv. res. 2005; 31(4):418-429.

6. Monteiro CFS, Dourado GOL, Graça Júnior CAG, et al. Mulheres em uso prejudicial de bebidas alcoólicas. Esc. Anna Nery. 2011; 15(3):567-572.

7. Silva MGB, Lyra TM. O beber feminino: socialização e solidão. Saúde debate. 2015; 39(106):772-781.

8. Ronzani TM, Ribeiro MS, Amaral MB, et al. Implantação de rotinas de rastreamento do uso de risco de álcool e de uma intervenção breve na atenção primária à saúde: dificuldades a serem superadas. Cad. Saúde Pública. 2005; 21(3):852-861.

9. Babor TH, Higgins-Biddle JC, Saunders JB, et al. AUDIT - Teste de Identificação de Problemas Relacionados ao Uso de Álcool: roteiro para Uso em Atenção Primária. Ribeirão Preto: PAI-PAD; 2008. 
10. Moretti-Pires RO, Corradi-Webster CM. Adaptação e validação do Alcohol Use Disorder Identifi cation Test (AUDIT) para população ribeirinha do interior da Amazônia, Brasil. Cad. saúde pública. 2011; 27(3):497-509.

11. Triola MF. Introdução à estatística. 10. ed. Rio de Janeiro: LTC. 2008; 682 p.

12. Guimarães VV, Florindo AA, StopaI SR, et al. Consumo abusivo e dependência de álcool em população adulta no Estado de São Paulo, Brasil. Revista Brasileira de Epidemiologia. 2010; 13(2):314-32.

13. Reitas ICM, Moraes SA. Dependência de álcool e fatores associados em adultos residentes em Ribeirão Preto, São Paulo, Brasil, 2006: Projeto OBEDIARP; Alcohol addiction and associated factors in adults in Ribeirão Preto, São Paulo State, Brazil, 2006: the OBEDIARP Project. Cad. Saúde Pública. 2011; 27(10):20212031.

14. Jomar RT, Abreu AMM, Griep RH. Padrões de consumo de álcool e fatores associados entre adultos usuários de serviço de atenção básica do Rio de Janeiro, RJ, Brasil. Ciênc. Saúde Colet. 2014; 19(1):27-38.

15. Rodríguez CC, Biset AED, Mayeta YB. Factores de riesgo de cáncer de mama em mujeres pertenecientes a um consultorio médico del centro urbano "José Martí”. Medisan. 2013; 17(9):4089-4095.

16. Segri NJ, Francisco PMSB, Alves MCGP, et al. Práticas preventivas de detecção de câncer em mulheres: comparação das estimativas dos inquéritos de saúde (ISA - Capital) e vigilância de atores de risco e proteção para doenças crônicas por inquérito telefônico (VIGITEL - São Paulo). Rev. bras. epidemiol. 2011; 14(1):31-43.

17. Simeão SFAP, Landro ICR, Conti MHS, et al. Qualidade de vida em grupos de mulheres acometidas de câncer de mama. Ciênc. Saúde Colet. 2013; 18(3):779788.

18. Souza MM, Winnikow EP, Moretti GP, et al. Taxa de mortalidade por neoplasia maligna de mama em mulheres residentes da região carbonífera catarinense no período de 1980 a 2009. Ciênc. Saúde Colet. 2013; 21(4):383-390.

19. Magnabosco MB, Formigoni MLOS, Ronzani TM. Avaliação dos padrões de uso de álcool em usuários de serviços de Atenção Primária à Saúde de Juiz de Fora e Rio Pomba (MG). Rev. Bras. Epidem. 2007; 10(4):637-647.

20. Oliveira MC, Ronzani TM. Estigmatização e prática de profissionais da APS referentes ao consumo de álcool. Psicol. ciênc. prof. 2012; 32(3):648-661.

21. Miccas FL, Batista SHSS. Educação permanente em saúde: metassíntese. Rev Saúde Pública [internet]. 2014; 48(1):170-185. [acesso em 2016 maio 19] Disponível em: www.scielo.br/scielo.php?script=sci_ar ttext\&pid=S0034-89102014000100170.

22. Fonseca CJB. Conhecendo a redução de danos enquanto uma proposta ética. Psicol. \& Saberes. 2012; 1(1):11-36.

23. Brasil. Decreto ${ }^{\circ}$ 9.761, de 11 de Abril de 2019. O presidente da república, no uso da atribuição que lhe confere o art. 84, caput, inciso VI, alínea "a”, da Constituição e tendo em vista o disposto na Lei ${ }^{\circ} 11.343$, de 23 de agosto de 2006. Diário Oficial da União. 12 Abr 2019.

Recebido em 28/12/2018

Aprovado em 07/06/2019

Conflito de interesses: inexistente

Suporte financeiro: não houve 